\title{
Prophylaxe nicht zu früh absetzen
}

\begin{abstract}
Männer und Frauen wurden nach aktuellen Zahlen des Statistischen Bundesamtes im Jahr 2006 wegen einer Thrombose stationär behandelt - davon waren 26.868 Frauen. Das waren immerhin 7587 Patienten weniger, die einen stationären Aufenthalt wegen einer Thrombose brauchten, als noch im Jahr 2000.

Auch die durchschnittliche Verweildauer sank innerhalb dieser Zeitspanne von 10,7 auf 8,7 Tage.
\end{abstract}

Krankenhausdiagnosestatistik des Statistischen

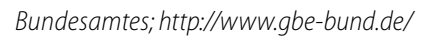

\section{Infos im Internet}

Detaillierte Informationen zur Prophylaxe von Venenthrombosen bietet die S3-Leitlinie der Deutschen Gesellschaft für Angiologie und weiterer Fachgesellschaften auf: http://leitlinien.net/

Informationen für Patienten und Interessierte bietet die kostenlose Broschüre „Thrombose - Vorbeugen und behandeln" der Deutschen Gesellschaft für Angiologie - Gesellschaft für Gefäßmedizin e. V. zum Download:

http://www.dga-gefaessmedizin.de

Regelmäßige Gymnastik hilft, bei langen Flugreisen Thrombosen vorzubeugen. Anleitung gibt die kostenlose App iFlySafe. Sie enthält 15 verschiedene Übungen, die Reisende im Sitzen und Stehen ausführen können: http://itunes.apple.com/app/ ifly-safe/id347315048?mt8

Viele praktische Tipps zur Diagnostik und Therapie bei Venenerkrankungen findet man darüber hinaus auch auf der Homepage der Deutschen Venen-Liga unter: www.venenliga.de
Das Rückfallrisiko bestimmt größtenteils die Dauer der Antikoagulation. Das richtig einzuschätzen, ist eine Herausforderung.

Die Entscheidung über die Therapiedauer richtet sich laut aktueller S2-Leitlinie neben der Lokalisation der TVT vor allem nach deren Genese. Bei einer sekundären Thrombose mit einem transienten Risikofaktor ist das Rückfallrisiko gering und die Antikoagulation kann unabhängig von der Ausdehnung der Thrombose nach drei Monaten beendet werden, rät Professor Sabine Eichinger, Universität Wien [1].

\section{Rückfallrisiko steigt auf $10 \%$ pro Jahr}

Als transiente Risikofaktoren gelten nach den Leitlinien des American College of Chest Physicians eine Operation, ein Klinikaufenthalt oder eine Gipsimmobilisation innerhalb der letzten drei Monate sowie eine Östrogentherapie, Schwangerschaft und längere Reisen. Fehlt ein solcher Faktor, steigt das Rückfallrisiko auf $10 \%$ pro Jahr. Diesen Patienten wird eine Antikoagulation mit Vitamin-K-Antago- nisten empfohlen, vorausgesetzt sie haben kein erhöhtes Blutungsrisiko, sind also unter 65 Jahre, weder Diabetiker noch nierenkrank und haben keinen Hirninfarkt oder Magen-Darm-Blutung in der Anamnese [2].

\section{Nach sechs Monaten erneut prüfen}

Nach aktueller Datenlage sollte jedoch auch bei Patienten mit idiopathischer Thrombose nach sechs Monaten die Notwendigkeit der Antikoagulation überprüft werden, wie Professor Bruno Geier vom Krankenhaus Bethanien in Moers betont [2].

Lassen sich zu diesem Zeitpunkt in der Duplexsonografie keine residuellen Thromben nachweisen und sind die DDimere normwertig, besteht laut PROLONG- und PROLONG-PLUS-Studie sowie DACUS- und Extended-DACUS-Studie ein sehr geringes Rezidivrisiko, und die Antikoagulation kann beendet werden. Andernfalls ist die Therapie weitere sechs Monate fortzuführen und danach erneut zu kontrollieren.

(dk)

[1] Hämostaseologie 2013, online 29. Mai;

[2] Gefäßchirurgie 2013, 18: 41

\section{Sichere Auswahl}

\section{Antikoagulieren in der Schwangerschaft}

In der Schwangerschaft ist das Repertoire der zur Prophylaxe und Therapie einsetzbaren Antikoagulanzien eingeschränkt.

Darf doch der Wirkstoff weder die Plazentaschranke passieren noch mit Blick auf die Geburt schlecht steuerbar sein. Niedermolekulare Heparine und der Faktor-Xa-Hemmer Fondaparinux er- füllen diese Anforderungen und sind Mittel der Wahl in der Schwangerschaft [1]. Schwangere mit Mitralklappenersatz profitieren allerdings, wenn nach Abschluss der Organogenese wieder auf Vitamin-K-Antagonisten umgestellt wird [2]. 